ISSN 2767-3901

\title{
Study of Quantization of Space-Time Explains Matter and Its Aspects
}

\author{
International Journal of Theoretical \& Computational Physics
}

Research Article

George Gerhab

Hellertown, USA

\section{*Corresponding author}

George Gerhab

Hellertown, USA.

Submitted : 1 Nov 2021 ; Published : 23 Dec 2021

\begin{abstract}
By quantizing space-time in such a way that I can calculate all of the constants of physics and derive and explain every equation therein.

The magnetic field appears when the electric field seems to travel faster than c. The same way an "anti-gravity" perpendicular field appears, totally depended on relative velocities, since the electric and gravitational forces are made of the micro field lines and are different aspects of the same force [1].

The "gravitational" perpendicular force can be repellant, when masses travel in the same direction, (massive distant galaxies repel enlarging the universe, new current mass just being released) and it can be attractive (opposite spiral arms moving in opposite directions, combined with a super-massive black hole).
\end{abstract}

The recent detection of gravity waves, is clear evidence for all of this since these waves need a perpendicular field, like electro-magnetic waves.

The magnetic style of the perpendicular field, at extremely high can cause matter and dark to interact

Keywords: Quantization of Space Time, Magnetic Style Perpendicular Gravity Field, Anti-gravity, Interaction of Matter with Dark Matter.

\section{Introduction}

The electron is, by far, the most important particle known. This tiny electric particle is responsible for every bio-chemical reaction and structure in our body as well as every chemical reaction that happens anywhere and it is also the tiny electric messenger for our computers and smartphones.

The electron has been known for over 100 years and is the most studied particle there is and, yet, no one knows what it is.

Perhaps, the reason for not knowing is that both scientists and people consider space and time to be continuous. Everyone knows intuitively what space and time are. Space is the place where objects move and time is how long it takes the objects to move. Since there are no jumps in time or space, they are considered continuous.

However, the electron cannot exist in a continuous space-time. Scientists have to renormalize (ie,fudge) their equations to account for the electron's apparently infinite charge and mass by infinitely screening them with virtual pairs of particles. This should say something besides, "?”.

The electron can only exists in a universe where both space and time are quantized.
Quantization of space means that no two points can get closer than a certain quantized distance, call this distance $\mathrm{x}_{0}$. All distances have to be whole numbers of this distance. Quantization of time means that time progresses in steps of a certain amount of time, call this amount of time $t_{0}$, rather than proceeding continuously as we believe. All time amounts must be whole numbers of the time $t_{0}$.

Space-time may consist of an infinite ocean of randomly appearing and disappearing pairs of points. One point of each pair jumps backward in time while the other point jumps forward in time. They blink back to zero in time and disappear in $t 0$ amount of time, thus preserving time quantization. The two points are exactly $\mathrm{x} 0$ distance apart which preserves space quantization. A point is a singularity which has no dimensions or structure to it. Points, I believe, are the only thing that can exist in the universe, because, if anything else did, with some type of structure, the immediate question would be, "What is it made of?". This question cannot be answered.

These virtual pairs of points build up space-time [2]. The points are not in space-time, they are space- time. The points in these virtual pairs must always be exactly $\mathrm{x} 0$ distance apart. Any other distance would disobey the very structure of spacetime itself. 
Two points of quantized space-time must share their time. That is, if one point's time jumps back to zero, another point's time (x0 away) must jump forward to zero, in the opposite direction, then the points disappear. Virtual space-time points disappear when two points share time in an integral step, in opposite directions (entanglement?). The integrity of the elementary unit of time cannot be violated. It is exactly the same everywhere, no matter what reference frame.

This sharing of time could be the mysterious effect of entanglement and, since the two points sharing time do not have to be the two virtual points that occurred together, entanglement would be a universal property of quantized space-time which could connect all of space-time throughout infinity.

The space quantization is created by the elementary time unit by the equation $\mathrm{x}_{0}=\mathrm{ct}_{0}$ where $\mathrm{c}$ is a space-time constant. Since the integrity of space-time cannot be violated, $\mathrm{c}$ is a universal constant. In natural units, $\mathrm{c}=1$, and time and space are one and the same thing. With space quantization, only equilateral triangles are possible and the myriad of virtual space-time points creates the 3 -D effect we are familiar with but any point in a $4^{\text {th }}$ dimensional space would not be possible cause it would have to violate the integrity of quantized space-time.

\section{The Singleton Point as The Particles of Matter} $+$

Now consider a singleton point, *

Which has time but no paired point to share its time with in order to disappear. It cannot move anywhere and would appear stuck. But with the vast amounts of virtual pairs constantly appearing and disappearing, one pair will certainly come close enough in order that the singleton point can share its time with one of the points of the virtual pair and disappear. The other point of the virtual pair becomes a new singleton point.

$\begin{array}{ll} & { }^{*+} \\ & \text { Virtual Pair } \\ \text { Singleton } & { }^{*} \text { - all distance are } \mathrm{X}_{0}\end{array}$

The negative time singleton point shares its time with the positive point of the virtual pair. These two points disappear leaving the negative time point as the new singleton point. In this way, the singleton point zips around, at random, at the speed of light and becomes matter. The equation for the random walk problem and Schroedinger's equation are identical except for the ih's.

$\mathrm{O}$

$$
\text { O * new singleton point }
$$

The singleton point has now moved a distance of $\mathrm{x} 0$ in a time $\mathrm{t}_{0}$ at the speed of light. Such singleton points move over $10^{44}$ times every second.

The only other way that this singleton can move is this:

$$
*_{+} \quad *_{-}
$$

$*$

\section{Singleton -}

Since all points must be $\mathrm{x} 0$ apart, they can only form equilateral triangles, and the only angle that can exist is $60 \mathrm{o}$. When the singleton point shares time with the + virtual point and disappears, we have.

$$
\mathrm{O} \quad *_{\text {- new singleton }}
$$

\section{$\mathrm{O}$}

The new singleton point has moved at an angle of $60^{\circ}$, the only angle, besides zero, that the singleton point can travel. Since $\cos 60^{\circ}=1 / 2$, this is the reason that spin is $1 / 2$.

The singleton point travels randomly at the speed of light within a small volume, since the virtual pairs randomly appear close to the singleton point. According to the random walk problem, this small volume expands (the differential equation for the random walk problem is nearly the same as Schroedinger's equation)

Since the new singleton point has suddenly appeared, it tries to share time with the virtual pair directly in front of it but it cannot since the new singleton would have traveled a distance $2 \mathrm{x}_{0}$ in a time $\mathrm{t}_{0}$, this shock imparts a small "tug" to this virtual pair which cannot "move" but instead gains a small amount of energy. This virtual pair does not disappear (since it has a small amount of energy) but, instead, travels outward at the speed of light through the array of virtual pairs. This is a small electric line. There are over $10^{44}$ of these small field lines being created every second by the electron.

When these small field lines get close enough to another, distant, electron, a force on this second singleton point is the result.

Each of these micro field lines that do get close enough must change the overall average velocity of the singleton point "package". This change must be permanently recorded within the "package", close enough to the singleton point, in order to occasionally influence its motion. Polarization of space-time creating etches as a result of these lines may be the method. The singleton point must travel by all of them (the history of the particle) in order to average out the overall average velocity.

\section{Micro-Field Lines and The Electric Force}

In order to calculate this force, what's first needed is to know how many tiny field lines are generated by the first electron. Since a field line is created every $t_{0}$ amount of time since $t_{0}$ is the elementary unit of time, the total number of lines generated every second is 1 divided by $t 0$ or $1 / t_{0}$. 
The next thing to know is how far away the second electron is since the field lines spread out over space hence there are fewer and fewer of them the further you get away from the first electron. The field lines spread out over the spherical area $4 \pi r^{2}$, hence the number of field lines that pass through a unit area at a distance $r$ is:

$$
\frac{1 / \text { to }}{4 \pi r^{2}}
$$

These field lines must get close enough to the second singleton point to increase the elementary time unit slightly so that the singleton point can travel a slightly larger distance in the same amount of time as measured by a stationary observer, therefore creating acceleration, since its velocity as far as the stationary observer is concerned in order to create a force. In a quantized space "close enough" means exactly $\mathrm{x}_{0}$ and the area near the singleton point that these field lines must pass through is $\left(\mathrm{x}_{0}\right)^{2}$. The force from these electric lines on the second electron is the number of these lines that pass through this area. The equation for this force is:

$$
\frac{\left(1 / t_{0}\right)\left(x_{0}\right)^{2}}{4 \pi r^{2}}
$$

The equation for the force between two electrons was experimentally determined by Coulomb around 1800 and it has been used ever since. It is:

$$
\frac{e^{2}}{4 \pi \varepsilon_{0} r^{2}}
$$

Where e is the elementary charge of the electron which is squared since the two electrons involved contribute charge and $\varepsilon 0$ is called the permittivity of free space and is a constant of free space.

Since (the elementary spacial distance) equals (the speed of light) times (the elementary time unit) or $\mathrm{x}_{0}=\mathrm{ct}_{0}$ is the relationship between space and time quantization. With this relationship, we have:

$$
\left(\mathrm{x}_{0}\right)^{2}=\left(\mathrm{ct} \mathrm{t}_{0}\right)^{2}=\mathrm{c}^{2} \mathrm{t}_{0}{ }^{2} \text { and }\left(1 / \mathrm{t}_{0}\right) \mathrm{c}^{2} \mathrm{t}_{0}{ }^{2}=\mathrm{c}^{2} \mathrm{t}_{0}
$$

Now if both equations for the electric force were set equal to each other it would look like:

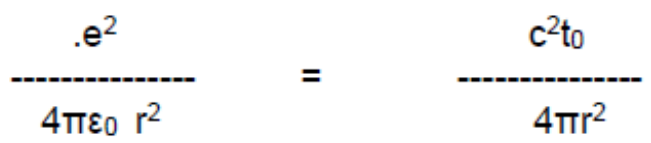

And, if the two equations are equal, then the following relationship must hold:

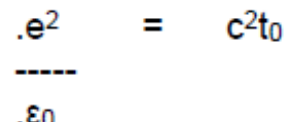

The speed of light is related to space and time using the following equation involving the constants of free space $\mu 0$ (the permeability of free space) and $\varepsilon_{0}$

\section{$c^{2}=1$ \\ $\varepsilon_{0} \mu_{0}$}

Now, from the previous equation, substituting for $\mathrm{c}$, we have:

$\begin{array}{lc}. e^{2} & t_{0} \\ -\varepsilon_{0} & =\end{array}$

Then, solving for the electric charge e, we have:

.e $\quad=\quad\left(\mathrm{t}_{0} / \mu_{0}\right)^{1 / 2}$ where the $1 / 2$ power means square root and which mathematically relates the elementary unit of charge, $\mathrm{e}$, to the elementary unit of time, $\mathrm{t}_{0}$.

From the measured value of $\mathrm{e}=1.602177 \times 10^{-19} \mathrm{C}$ where $\mathrm{C}$ is the charge unit Coulomb.

The calculated value of $t_{0}$ is $3.2343 \times 10^{-44}$ seconds or $(3 / 5) t_{p}$ where $t_{p}$ is the Planck time.

For continuous time, $\mathrm{t}_{0}=0$, so $\mathrm{e}=0$ and there can be no electron.

The elementary time unit, $\mathrm{t}_{0}$, that was found from the calculation was exactly three-fifths times the Planck time (considered by most to be the smallest quantity of time) $3 / 5$ is not an arbitrary fraction. $3 / 5$ is part of the 3-4-5 triangle which is the only triangle that has integral (whole number) steps on all three sides which goes along perfectly with the quantization of time. If time does progress in integral steps, then time would have to travel along the path of one of the sides of this triangle.

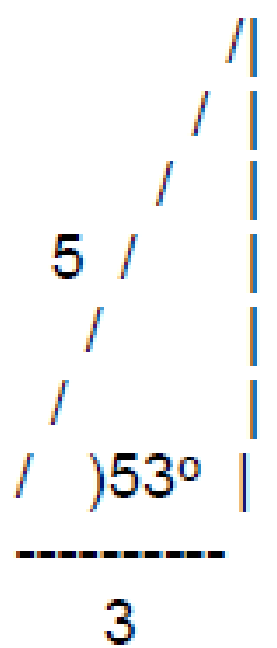

3 is our side of time and our time progresses at a rate $(3 / 5)$ times the Planck time which represents the $(5 / 5)$ side of the triangle. The two sides $(5 / 5)$ and $(4 / 5)$ may be considered dark matter since their different time unit would mean that they cannot interact with our matter.

(more information about this theory can be found in http:// www.sciencepub.net/academia Vol 5 \#9 2013 5(9) titled "The Derivation of Dirac's Equation" by George Gerhab) 
Energy of The Micro-Electric Field Lines

Each tiny electric field line that is created by the singleton point has a small amount of energy associated with it. The two points of a virtual pair become "unsynchronized" because the point next to it, which will suddenly become the singleton point, lasts longer (once as virtual and once as real) and can transfer an impulse to the virtual pair in front. The point cannot disappear with the point next to it because the new singleton point would move $2 \mathrm{x}_{0}$ in a time $\mathrm{t}_{0}$ meaning it would be traveling twice the speed of light which is not allowed.

This energy takes the form of the "unsynchronization" of the pair's time vectors and the virtual can no longer disappear and becomes a micro-electric field line. Consider the following diagram:

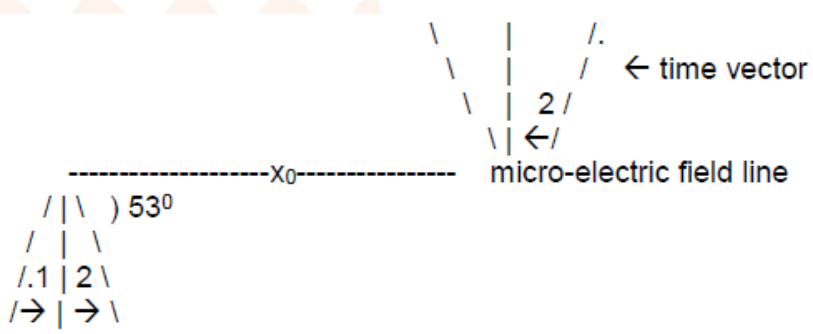

The bottom time vector starts at $53^{\circ}$ and goes backward in time, initially, which would make it a positron, since it is $180^{\circ}$ out of phase with the electron. If the pair is knocked out of synch, then step 1 on the bottom would not correspond to step 2 on top and these points would not be able to disappear, but rather travel outward, possibly giving its impulse to another charged particle.

The impulse given to this pair would have to depend upon the time, $\mathrm{t} 0$, which the time that the impulse is given, and the strength of the force giving it this impulse, namely $\left(4 \pi \varepsilon_{0}\right)^{-1}$. Since there are two points involved, these would have to be squared:

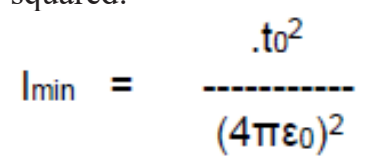

The equation for the Plank time is:

$. t_{\mathrm{p}}=(\mathrm{Gh} / \mathrm{c} 5)^{1 / 2}$ while for our elementary unit of time, we have $t_{p}=(5 / 3) t_{0}$ hence

$. \mathrm{t}_{0}^{2}=(3 / 5)^{2}\left(\mathrm{Gh} / \mathrm{c}^{5}\right)$ inserting this into the equation for Imin yields:

$\operatorname{Imin}=(3 / 5)^{2}\left(\mathrm{Gh} / \mathrm{c}^{5}\right)(4 \pi \varepsilon 0)^{-2}$ since $\mu 0^{2} \varepsilon 0^{2}=1 / \mathrm{c}^{4}$ and $\mathrm{h}=\mathrm{h} / 2 \pi$ and $\mu_{0}=\mathrm{t}_{0} / \mathrm{e}^{2}$

Where $\mathrm{G}=$ the Gravitational Constant $=6.672 \times 10^{-11} \mathrm{~N}-\mathrm{m}^{2} / \mathrm{kg}^{2}$ $. \mathrm{e}=$ elementary unit of charge $=1.602 \times 10^{-19} \mathrm{C}$

$. c=$ speed of light $=3 \times 10^{8} \mathrm{~m} / \mathrm{s}$

$. \mu 0=4 \pi \times 10^{-7} \mathrm{~N}(\mathrm{~s} / \mathrm{C})^{2}$ and taking the $4 \pi$ out into $\mathrm{I}_{\min }$ yields:

$$
\left.I_{\min }=4 \pi h x_{0}(3 / 5)^{2} \frac{G \times 10^{-7}}{32 \pi^{3} c^{2} e^{2}}\right)=4 \pi h x_{0}(\pi / 3)
$$

The calculation $(3 / 5)^{2}\left(\frac{G \times 10^{-7}}{32 \pi^{3} \mathrm{c}^{2} \mathrm{e}^{2}}\right)=\pi / 3$ has a great deal of

implication, $\pi / 3$ is

The exact number of radians in 600 which means that in Imin this would be $(\pi / 3)_{\mathrm{x} 0}$ which would form an arc between points, keeping the transfer of the impulse, Imin, exactly the distance $\mathrm{x} 0$ away from all other points in space-time. This seems to mean that 5 very well known physical constants are "conspiring" to make sure that everything in space is exactly $\mathrm{x} 0$ away from each other and that the only angle in space allowable is $60^{\circ}$.

The Actual calculation is 1.04798 while $\pi / 3=1.047194$, which I think is pretty close.

Hence $\mathrm{I}_{\min }=4 \pi \mathrm{h}(\pi / 3)_{\mathrm{x} 0}$

Since this calculation is so accurate and so meaningful, it cannot be coincidental. As such, it lends validity to the elementary time unit since $(3 / 5)^{2}$ is part of the calculation as well as the equation $\mathrm{t}_{0}=\mathrm{e}^{2} \mu 0$, which also was used in this calculation.

The units of this is $\mathrm{m}^{2} / \mathrm{C}^{4}$ which, actually, makes sense since two virtual pairs (having time which is associated with each point consequently charge) hence an area $\left(\mathrm{m}^{2}\right)$ can indeed be associated with the charge unit to the fourth power.

\section{The Magnetic Field and Special Relativity}

The micro-electric field line must not be seen as traveling faster than light from any inertial reference frame.

If an inertial reference frame is traveling at a speed, $v$, in the opposite direction that the micro-line is, this would look like the micro-line could be traveling faster than light and endangering the integrity of quantized space time.

Instead of the micro-electric line being seen as traveling faster than light, another field arises, perpendicular to the E-line and dependent on the velocity, $\mathrm{v}$, in order to preserve the integrity of space-time. This field is the well known magnetic field. Graphically:

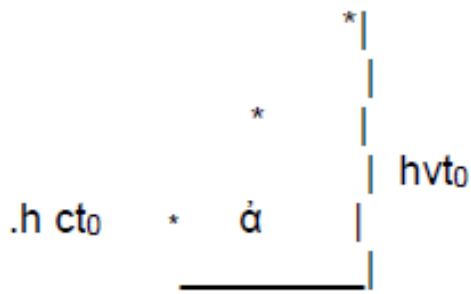

\section{$E$}

Where $\sin \dot{\alpha}=\left(\right.$ hvt $\left._{0}\right) /\left(\right.$ het $\left._{0}\right)=\mathrm{v} / \mathrm{c}$

So that the left side of the law of tangents becomes:

$$
1+\frac{\sin ^{2} \dot{\alpha}}{\cos ^{2} \dot{\alpha}}=1+\frac{\sin ^{2} \dot{\alpha}}{1-\sin ^{2} \dot{\alpha}}=1+\frac{(v / c)^{2}}{-1-(v / c)^{2}}=\frac{1}{1-(v / c)^{2}}
$$


In order to get the real velocity from the magnetic field velocity, a rotation of $90^{\circ}$ is needed. Hence $\dot{\alpha} \rightarrow \dot{\alpha}-90^{\circ}$ and $\cos (90-\dot{\alpha})=\sin \dot{\alpha}$, so that the right side of the law of tangents becomes $\sec ^{2}=1 / \cos ^{2}(90-\dot{\alpha})=1 / \sin ^{2} \dot{\alpha}=(\mathrm{p} / \mathrm{mv})^{2}$, where mv is what the momentum would be in a continuous space-time and infinite speed limit, and $p$ is the actual momentum as seen by an inertial reference frame that sees the particle traveling at speed v. Hence, the law of tangents then becomes:

$1+\tan ^{2} \dot{\alpha}=\frac{1}{1-v^{2} / c^{2}}=\sec ^{2}(90-\dot{\alpha})=\frac{p^{2}}{(m v)^{2}} \rightarrow p=\frac{m v}{\sqrt{\left(1-v^{2} / c^{2}\right)}}$

The magnetic field, $\mathrm{B}$, is related to the electric field, $\mathrm{E}$, by $\mathrm{B}=$ $\mathrm{Ex}(\mathrm{v} / \mathrm{c})$ and the magnetic force is related to the electric force by: $\mathrm{F}_{\text {mag }}=\mathrm{F}(\mathrm{v} 1 \mathrm{x} \mathrm{v} 2) / \mathrm{c}^{2}$. Using the equation for the electric derived earlier:

$\mathbf{F}_{\text {mag }}=\left(c^{2} t_{0}\right) /\left(4 \pi r^{2}\right)\left(\mathbf{V}_{1} \times \mathbf{V}_{2}\right) / c^{2}=t_{0}\left(\mathbf{V}_{1} \times \mathbf{V}_{2}\right) /\left(4 \pi r^{2}\right)=\mu_{0} e^{2}\left(\mathbf{v}_{1} \times \mathbf{V}_{2}\right) /\left(4 \pi r^{2}\right)$

Since $\mathrm{t}_{0}=\mu_{0} \mathrm{e}^{2}$

\section{The Gravitational Field}

As stated earlier, when a singleton point skips a distance $\mathrm{x} 0$, it creates an Imin micro-field line that travels outward at the speed of light and creates the electric force. As also stated, these micro-field lines sweep over a distance of $(\pi / 3) \times 0$ to maintain the elementary distance from the virtual space-time points.

The volume created by a pair (one from a + charge and one from a - charge) would be: $(\pi / 3)^{2} \times 0^{2}(2 \pi \times 0)$ since there would be two $(\pi / 3)$ sides and one $2 \pi$ circumference.

These larger elementary volumes would be occurring on a temporary basis at a frequency that depends on mass and distance.

The number of Imin micro-lines emitted by a massive object and occurring at a distance $r$ is: $B=$ \#field lines at $r=(1 / c)(M /$ $\mathrm{m} 0) \mathrm{N}$

$\mathrm{N}=1 / \mathrm{t}_{0}=$ \#lines emitted by one charged particle, $\mathrm{t}_{0}$ is the elementary unit of time, $\mathrm{m}_{0}$ is the elementary unit of mass (nucleon + electron) $\mathrm{c}$ is the distance it travels in one second, but we want the number at the distance $r$.

Since the field lines created a larger volume, the volume that a particle could see would be, for a thin shell around a mass would be:

$\mathrm{A}=$ shell volume/elementary volume unit $=$ \#elementary volumes $=\frac{(\pi / 3)^{2} 2 \pi\left(4 \pi r^{2}\right) x_{0}}{. x_{0}^{3}}$

$$
\frac{A}{B}=\frac{M(9 \times 0)}{r^{2}\left(8 \pi^{4} m_{0}\right)}=\frac{M}{r^{2}}
$$

The Impulse and Wave Equations

The energy given by this electric micro-impulse, Imin, depends on the uncertainty of the singleton point, which is traveling at the speed of light within a distance of $\Delta x$, and, as such, its position can never be exactly determined. The energy of these micro- impulses is determined by how close, $\Delta \mathrm{x}$, they are to each other and the singleton point. The total energy of the field is multiplied by how many movements the singleton point has per second, $\mathrm{N}=1 / \mathrm{t}_{0}$. Hence, the total uncertainty of the energy of the field is:

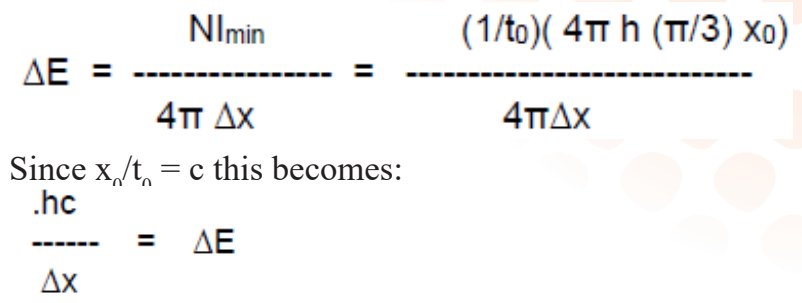

Since $\Delta \mathrm{x}=\mathrm{c} \Delta \mathrm{t}$ and $\Delta \mathrm{E}=\Delta \mathrm{p}$, , so this becomes:

$\frac{. h c}{\Delta x}=\Delta p c$ or $h \sim \Delta p \Delta x$ and $\frac{h \mathrm{c}}{\mathrm{c} \Delta \mathrm{t}}=\frac{\mathrm{h}}{\Delta \mathrm{t}}=\Delta \mathrm{E}$ or $\mathrm{h} \sim \Delta \mathrm{E} \Delta \mathrm{t}$

If $\Delta \mathrm{x}$ can be considered a wavelength, $\lambda$, and $1 / \Delta \mathrm{t}=\mathrm{f}$, a frequency, then these equations would become:

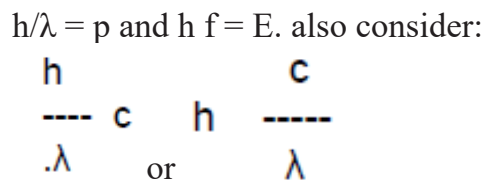

They seem to be the same, yet $\mathrm{h} / \lambda$ does not infer that the wave is traveling at the speed of light, whereas $\mathrm{c} / \lambda$ does. $E=p c$ is the energy of the field which is traveling less than the speed of light, while $\mathrm{E}=\mathrm{hf}$ is the energy of the singleton point which is traveling at the speed of light. It seems that there are two waves simultaneously being carried out in the electron. A singleton point wave (governed by Dirac's equation) and a field wave consisting of all the micro-fields lines that have been saved, and became part of the particle's field (governed by the KlineGordon equation).

\section{The Wave}

The time motion of the time vector of the singleton point is:

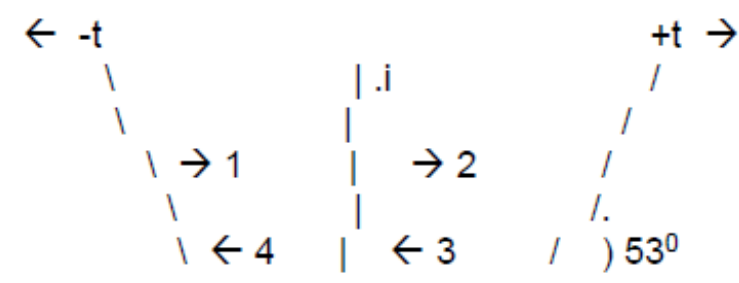

The electron starts at negative $\mathrm{t} 0 \cos 530$ or $-(3 / 5) \mathrm{t}_{0}$ and initially moves forward in time for two time clicks to $+(3 / 5) \mathrm{t}_{0}$, then it moves backward in time ( $3 \& 4)$ for two time clicks, then it moves back and forth, indefinitely. Each time motion requires a separate time function:

$\Psi=\quad \begin{aligned} & \Psi_{1} \\ & \Psi_{2} \\ & \Psi_{3} \\ & \Psi_{4}\end{aligned}$


Each of these functions can have either + or $-1 / 2$ spin since the only angle allowed by quantize space is $60 \mathrm{o}$ and the $\cos 60^{\circ}=1 / 2$, the exact spin of the electron [3].

These functions can change spin and order as such:

$$
\Psi=\quad \begin{array}{ll} 
& +\Psi_{1} \\
& -\Psi_{2} \\
& +\Psi_{3} \\
& -\Psi_{4}
\end{array}
$$

These functions can change places with the positive time movements.

The possibilities for the exchanges in the four columns is:

$$
\begin{aligned}
& 6+\psi 2-\psi 1 \\
& -\psi 1 \quad-\psi 2 \\
& \grave{\alpha} 1 \psi=-\psi 4 \quad \dot{\alpha} 3 \psi \quad=-+\psi 3 \\
& +\psi 3+\psi 4
\end{aligned}
$$

The $2^{\text {nd }}$ matrix has imaginary numbers in it, which means that the imaginary part of the wave function plays a real role in the solution ofthe Dirac function. Since $\operatorname{sine}(53)=\cos (37)$, It seems to me that $37^{\circ}$ is the phase factor for a part of dark energy, since it's the only other time phase factor that is possible in the 3-45 triangle which represents the only integral time movements that are possible if time moves in integral steps.

The other Dirac matrix, 6, has the reverse time functions reversed to the top two functions in the function column. This is more like a positron function which starts at $+\mathrm{t} 0$ and moves backward in time, $180^{\circ}$ out of phase with the electron.

With these Dirac matrix, the Hamiltonian becomes: $\alpha$

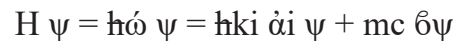

$$
\begin{aligned}
& \partial \psi \\
& \text {-.ih } \frac{-}{\partial t}=-. i h_{i}^{\alpha_{i}} \underset{\partial x_{i}}{--.}+m c b \psi
\end{aligned}
$$

This is Dirac's equation, mostly electron with dark matter and positron mixed in. The Muon and the Family of Particles A micro-field line is a + and a - point exchanging time rapidly and causing space-time to oscillate between + and - but cannot disappear because they've been given a slight "tug" of $\mathrm{I}_{\min }$. When two such sets of microline points come close enough (within a distance $\mathrm{x} 0$ which would occur in high energy collisions) two points, one from each pair, can exchange time and disappear, leaving the other two points separated by $2 \mathrm{x} 0$ and are free and can become enveloped into an electron's field, creating a three point muon. Two of the points eventually get close enough to exchange time which neutralizes the points and become neutrinos. The electron is left over.

The anomalies of the muon's magnetic moment and its anomaly of increasing the size of the proton can be explained by this explanation of the muon. Three points rather than one would have a different magnetic moment than the electron. While orbiting a proton, the two negative points of the three would be closer to the proton, enlarging it more than the electron would.

\section{Anti-Gravity}

Just like the magnetic field appears in order to keep the electric micro-lines from appearing to travel faster than light speed (which would affect the elementary units of time and space) , a magnetic like field appears to prevent the motion of the gravitational field density from traveling faster than light speed as well [4].

Gravitational objects traveling at some speed measured by an inertial reference frame must have a field perpendicular to the normal gravitational field. If two such gravitational objects are traveling in the same direction, according to a third inertial frame, they would seem to repel each other similar to the way currents in the same direction do. Only extreme speeds (distant galaxies traveling away from us at close to light speed.) would cause this repulsion.

Distant galaxies traveling close to light speed would have an enormous mass-energy $\left(E=\mathrm{mc}^{2} / \sqrt{ }\left(1-\mathrm{v}^{2} / \mathrm{c}^{2}\right)\right)$ and could repel all similar galaxies which would expand the space between them and drag the rest of the universe with it

When the universe began, matter was blown straight out, very near the speed of light, and the perpendicular repulsion of gravity would expand the universe suddenly.

In galaxies, one arm of the spiral is traveling in the opposite direction as the spiral arm on the opposite side of the center. In magnetism, two currents traveling in opposite direction attract. The two spiral arms would act in the same way, and give an addition attraction in the galaxies, not to mention the supermassive black holes that are found in each and everyone of them.

Electro-magnetic waves are formed by two forces that are perpendicular to each other caused by the fact that no force field can travel faster than light (inherent in the basic fabric of all space-time). Gravitational waves would indicate that there exists an anti-gravity force perpendicular to the normal gravity field, resulting from the fact that the gravity field, like the electric field, cannot travel faster than the basic speed within the fabric of all space-time.

In the beginning, when the universe blew up, matter was traveling outward at near this speed, and repelled each other and expanded the universe suddenly. The universe has been expanding ever since.

The recent measurement of gravity waves does now seem to verify this and it also seems to show that these forces are one in the same. 
Big Bangs

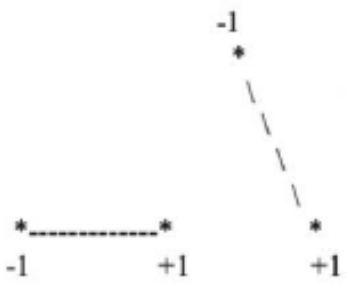

Two virtual pairs blink as shown, but, instead of the pairs blinking normally, the above -1 point tries to share time with two +1 points at the same time. Since only one elementary time unit is allowed during each integral step of time, this would be impossible, but in an extremely rare case only a fraction of the time unit would, because of entanglement, be exchanged and create partial charges, $-1 / 3,+2 / 3$. They are held together by entanglement gone wrong.

Since these points become real, and last a long time, they polarize space-time and the once highly unlikely event now occurs every $t_{0}$ seconds and $1 / t_{0}$ number of electrons and protons occur every second. When the points become real, they create extra space which causes the particles to fly out.

The other two types of matter (time phase angle $37^{\circ}$ and $0^{\circ}$ ) have a larger elementary time unit, (4/5) $t_{p}$ and $t_{p}$, they have a better chance at creation then our matter does, which is 1 chance in 4 . Also since the elementary field unit $\left(e=\sqrt{ }\left(t_{0} / \mu_{0}\right)\right)$ is larger for dark matter, it would have more mass. A ratio of 5 to 1 , dark over our matter, is not inconsistent with my theory.

\section{Gravity and The New Physics}

\section{The Creation of Magnetism}

In my previous paper " "The micro-field lines must not see to be traveling faster than light in any inertial reference frame.

If an inertial reference frame is traveling at a speed, $v$, in the opposite direction that the micro field line is, this would look like the micro-field line could be traveling could be traveling faster than light and endangering the integrity of quantum space-time.

Instead of the micro-electric line being seen as traveling faster than light, another field arises, perpendicular to the E-line and dependent on the velocity, $\mathrm{v}$, in order to preserve the integrity of space time. This field is the magnetic field"

\section{The Magnetic Style Gravity Field}

Then, in my paper [1] I proposed that gravity was caused by the + and - micro-field lines from a massive object. The micro-field lines travel in arcs of $\pi / 3$ which is 60 degrees, the only angle and quantized space time can have (calculated earlier) Consequently they form a larger elementary unit of volume than regular non-matter space can have which (I propose) cause other objects to be attracted to it I calculated the universal gravitational constant to a ratio of elementary space to elementary mass and got $6.68 \times 10^{-11}$.
Since (I propose) that the electro-magnetic and gravitational fields are from exactly the same thing (namely the motion of singleton points that make matter and create the micro-field lines and were separated from their pair at the time of the big bang) that gravity (I propose) would also have a perpendicular field, similar to the magnetic field.

Since all velocities of gravitational fields are very small, this perpendicular would be very small and hard to detect. But with massive galaxies traveling outward, with velocities(in the same direction) near light speed, it would result in a very strong repulsive gravity that could explain the expansion of the universe. If it is detected, then I am right.

The magnetic force is proportional to $\mathbf{q}_{1} \mathbf{v}_{1} X \mathbf{q}_{2} \mathbf{v}_{2}$ so the magnetic style gravity force should be proportional to $\mathbf{m}_{1} \mathbf{v}_{\mathbf{1}} \mathrm{X}$ $\mathbf{m}_{2} \mathbf{v}_{\mathbf{2}}$. But $\mathrm{mv}$ is momentum and the relativistic momentum is $\gamma \mathrm{mv}$, hence the perpendicular gravity field must be multiplied by $\gamma_{1} \gamma_{2}$ and when speeds become close to that of $\mathrm{c}$, this force can become extremely large. Since dark matter is also attracted by gravity at extremely high speeds, matter and dark matter could interact [1].

However, at low speeds, this perpendicular force of gravity would be so weak, that this perpendicular component of gravity would be almost undetectable. Satellites motion over time my exhibit this force. Large galaxies traveling in same direction at high speeds would strongly repel each other.

The 5th force breakthrough could, very well, be this perpendicular component of gravity at extremely high speeds. A + particle smashing into a - particle at near the speed of light would release pairs of + and - micro-field lines (gravity) and result in an extremely strong perpendicular gravity force. Ordinary and dark matter would be strongly attracted to each other and interact.

Entanglement may be a property of identical particles that eliminates the virtual pairs between them, so that, to them, they are always the elementary distance apart, no matter how far apart they seem to us.

\section{Conclusion}

My method of space-time quantization can explain what matter is and how it can appear in both wave and particle aspects, how both electro-magnetic and gravitation can be explained and both fields are the same.

\section{Competing Interests}

Author has declared that no competing interests exist. 


\section{References}

1. Quantizing space-time can explain matter and all of its aspects. The International Journal of Theoretical and Mathematical Physics.

2. George Gerhab (2013) The Derivation of Dirac's Equation article.sapub.org/10.5923.j.ijtmp.2 2013;5(9). Available:sciencepub.net/academia

3. Wikipedia, online encyclopedia (To refresh my mind on gravity equations)

4. David R. Lide, Editor in Chief. The Handbook of Chemistry and Physics. 1993-1994 edition (To look up the experimental values of the physical constants).

Copyright: ${ }^{0} 2021$ George Gerhab. MICHELINI. This is an open-access article distributed under the terms of the Creative Commons Attribution License, which permits unrestricted use, distribution, and reproduction in anymedium, provided the original author and source are credited. 